\title{
A RAMAN LIDAR WITH A DEEP ULTRAVIOLET LASER FOR CONTINUOUS WATER VAPOR PROFILING IN THE ATMOSPHERIC BOUNDARY LAYER
}

\author{
${\text { Masanori Yabuki }{ }^{*} \text {, Yuya Kawano }}^{2}$, Yusaku Tottori ${ }^{2}$, \\ Makoto Tsukamoto $^{2}$, Eiji Takeuchi ${ }^{2}$, and Toshitaka Tsuda ${ }^{1}$ \\ ${ }^{1}$ Research Institute for Sustainable Humanosphere, Kyoto University, Uji, Kyoto 611-0011, Japan \\ ${ }^{2}$ EKO Instruments Co., Ltd., Shibuya-ku, Tokyo 151-0072, Japan \\ *Email:yabuki@rish.kyoto-u.ac.jp
}

\begin{abstract}
A Raman lidar with a deep ultraviolet laser was constructed to continuously monitor water vapor distributions in the atmospheric boundary layer for twenty-four hours. We employ a laser at a wavelength of $266 \mathrm{~nm}$ and detects the light separated into an elastic backscatter signal and vibrational Raman signals of oxygen, nitrogen, and water vapor. The lidar was encased in a temperature-controlled and vibration-isolated compact container, resistant to a variety of environmental conditions. Water vapor profile observations were made for twelve months from November 24, 2017, to November 29, 2018. These observations were compared with collocated radiosonde measurements for daytime and nighttime conditions.
\end{abstract}

\section{INTRODUCTION}

Water vapor, one of the most variable atmospheric constituents, plays an important role in atmospheric processes such as the atmospheric energy budget and the global water cycle. The distribution of water vapor is associated with that of clouds and rainfall through the vertical stability of the atmosphere caused by a large amount of latent heat related to the phase changes of water. Therefore, water vapor is a key parameter in understanding the localized extreme weather events associated with severe weather disasters such as torrential rain and floods. Information on the spatiotemporal distribution of water vapor is highly beneficial for improving the accuracy of weather forecasts made by mesoscale numerical weather prediction models.

The Raman lidar technique is a wellestablished tool for measuring the water vapor mixing ratio in the atmosphere ${ }^{1}$. While most of the Raman lidar previously used for the monitoring and field observation of water-vapor distribution employ a laser wavelength of $355 \mathrm{~nm}$, Renaut et al. (1980, 1988) and Lazzarotto et al. (2001) demonstrated the Raman lidar with a $266 \mathrm{~nm}$ laser and a grating polychromator to conduct daytime observations during periods of high sky radiance ${ }^{2,3}$. The use of the deep ultraviolet wavelengths is convenient because of the low background noise during the daytime, since most of the solar radiation in the wavelength range below $300 \mathrm{~nm}$ is absorbed by the ozone layer in the stratosphere. Recently, the improved performance of UV optical components has led to the development of enhanced Raman lidar systems with a deep ultraviolet lidar. In this study, we constructed a Raman lidar by employing a laser at a wavelength of $266 \mathrm{~nm}$ and an interference-filter-based polychromator.

\section{METHODOLOGY}

\subsection{Water vapor profiles from Raman lidar measurements}

The water-vapor mixing ratio $m$ at range $R$ is obtained from the Raman backscatter signals of 
water vapor $P_{\lambda_{\mathrm{H}_{2} \mathrm{O}}}$, nitrogen $P_{\lambda_{\mathrm{N}_{2}}}$, and oxygen $P_{\lambda_{\mathrm{O}_{2}}}$ as follow:

$$
\begin{aligned}
m(R) & =K_{m} \frac{P_{\lambda_{H_{2} O}}(R)}{P_{\lambda_{N_{2}}}(R)} \\
& \times\left[\frac{P_{\lambda_{O_{2}}}(R)}{P_{\lambda_{N_{2}}}(R)}\right]^{\frac{\sigma_{O_{3}}\left(\lambda_{H_{2} O} O\right)-\sigma_{O_{3}}\left(\lambda_{N_{2}}\right)}{\sigma_{O_{3}}\left(\lambda_{N_{2}}\right)-\sigma_{O_{3}}\left(\lambda_{O_{2}}\right)}} \Delta T,
\end{aligned}
$$

where $K_{\mathrm{m}}$ is the calibration constant and commonly evaluated by comparing the results of an independent measurement (e.g., radiosonde) of the water vapor mixing ratio; $\sigma_{\mathrm{O}_{3}}\left(\lambda_{x}\right)$ is the ozone absorption cross section at Raman-shifted wavelength $\lambda_{x}$ of species $x$; and $\Delta T$ indicates the difference in transmission of each Raman-shifted wavelength due to Rayleigh and Mie scattering processes.

\subsection{System setup}

The experimental setup of the Raman lidar is shown in Fig. 1. We employed the fourth harmonic output (266 nm) of an Nd:YAG laser (Continuum Surelite III-10, USA) and operated with the pulse energy of $<50 \mathrm{~mJ}$. The beam divergence of the outgoing laser beam after passing the beam expander is $0.17 \mathrm{mrad}$. A custom-made Cassegrain telescope (Raymetrics, Greece) with a primary mirror diameter of $200 \mathrm{~mm}$ and a focal length of $1000 \mathrm{~mm}$ was used as the optical receiver. The backscattered radiation was separated by the dichroic beam splitters and the interference filters to elastic and inelastic wavelengths of $266 \mathrm{~nm}$ for the elastic scattering, and $277.5 \mathrm{~nm}, 283.6 \mathrm{~nm}$, and $294.6 \mathrm{~nm}$ for the vibrational Raman scattering of oxygen, nitrogen and water vapor molecules, respectively. Three photomultiplier tubes (PMT) were used for detecting each backscatter light (R9880U, Hamamatsu, Japan). The data acquisition was performed using a four-channel transient recorder (TR 20-16bit, Licel, Germany) to

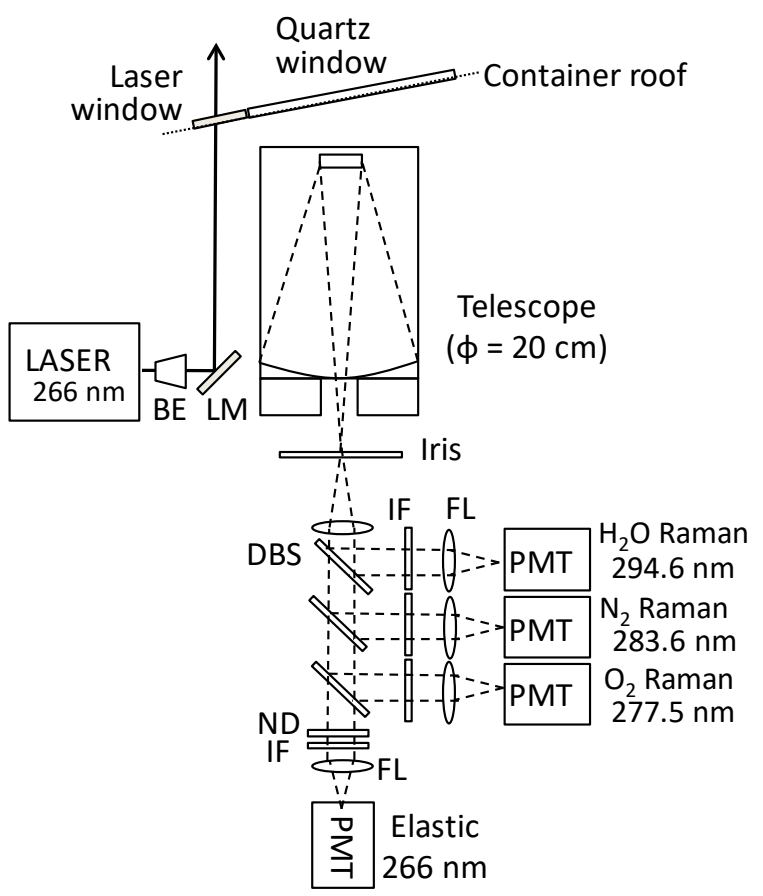

Figure 1. Schematic setup of water vapor Raman lidar. BE: beam expander, LM: laser mirror. FL: focusing lens; ND: neutral density filter; DBS: dichroic beam splitter; IF: interference filter; PMT: photomultiplier tube.

record the analog and photon counts in the signal pulses. The lidar was encased in a temperaturecontrolled and vibration-isolated compact container with laser and quartz windows for transmitting the laser beam and the scattered light from the atmosphere, respectively.

\section{RESULTS}

The Raman lidar container was installed at the Shigaraki MU radar observatory $\left(34^{\circ} 51^{\prime} \mathrm{N}\right.$, $136^{\circ} 06^{\prime} \mathrm{E}, 385 \mathrm{~m}$ a.s.1.) of the Research Institute for Sustainable Humanosphere (RISH), Kyoto University, Japan, from November 24, 2017, to November 27, 2018. The MU radar observatory is located in Shigaraki in the central part of the Kinki Region of Japan and is surrounded by a mixed hardwood-coniferous forest.

The temporal variation of the calibration constant was evaluated by comparison with 
collocated measurements from a radiosonde that was launched 21 and 14 times during the daytime and nighttime, respectively, during the year-long lidar observation period. The calibration constants of both analog and photon counting signals indicated a monotonic variation within $3.5 \%$ per year.

Water vapor mixing ratio profiles from the radiosonde and the Raman lidars for selected cases during nighttime and daytime are shown in Fig. 2. The trends shown by each observation agreed well up to about $1500 \mathrm{~m}$, which is the top of the atmospheric boundary layer despite the low water vapor mixing ratio in winter. The height resolutions were determined in the range of 30-150 m every 15 minutes in the atmospheric boundary layer, considering statistical errors calculated from the signals.
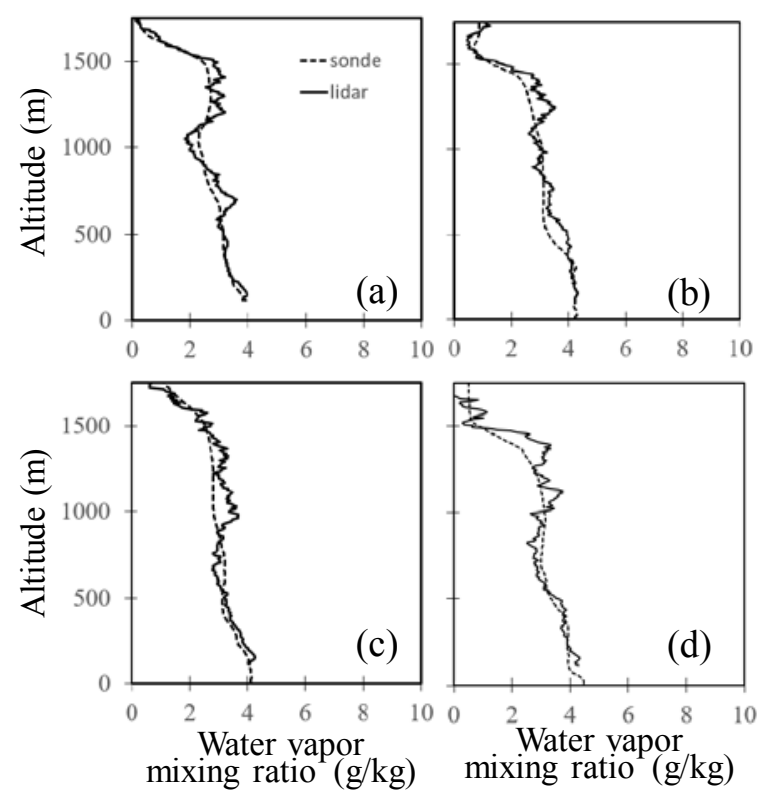

Figure 2. Water vapor mixing ratio profiles from the radiosonde (dashed line) and the Raman lidars (solid line) at Shigaraki, Japan at (a) 20:59-21:14 JST on December 22, 2017; (b) 01:15-01:30 JST; (c) 07:21-07:36 JST and (d) 10:41-10:56 JST on December 23, 2017. The range resolution of the Raman lidar changes in height from $30 \mathrm{~m}$ to $150 \mathrm{~m}$, considering the Raman signal statistics.
Figure 3 shows time-height cross sections of the water vapor mixing ratio profiles observed by the Raman lidar. The lidar worked well throughout the twelve months under air temperature variations between $-10{ }^{\circ} \mathrm{C}$ and $35^{\circ} \mathrm{C}$, except for the middle of the February and the beginning of the October during which time laser emission was stopped because of trouble with the air conditioner in the lidar container. In most cases where the observation range did not reach a height of $1500 \mathrm{~m}$, there were optically thick clouds and fogs at the lower height level. Red arrows in Fig. 3 correspond to the time of replacing the flash lamp of the laser. The variations of the calibration constant as well as the derived water vapor profiles were small before and after replacing the flashlamp every 2-4 months.

\section{CONCLUSIONS}

We have constructed a Raman lidar for observing long-term monitoring of water vapor mixing ratio profiles in the atmospheric boundary layer for twenty-four hours. The Raman lidar employs a laser at a wavelength of $266 \mathrm{~nm}$, characterized by the low background noise during daytime, and an interference-filter-based polychromator. The lidar was encased in a temperature-controlled and vibration-isolated compact container. We have demonstrated the potential of the developed lidar by collecting observations over a whole year at Shigaraki MU radar observatory in Japan. The lidar worked well throughout the twelve months. Water vapor mixing ratio profiles from the radiosonde and the Raman lidars agreed well up to about $1500 \mathrm{~m}$ which is the top of the atmospheric boundary layer. The variations of the calibration constant, as well as the derived water vapor profiles, were small before and after replacing the flashlamp every 2-4 months. 


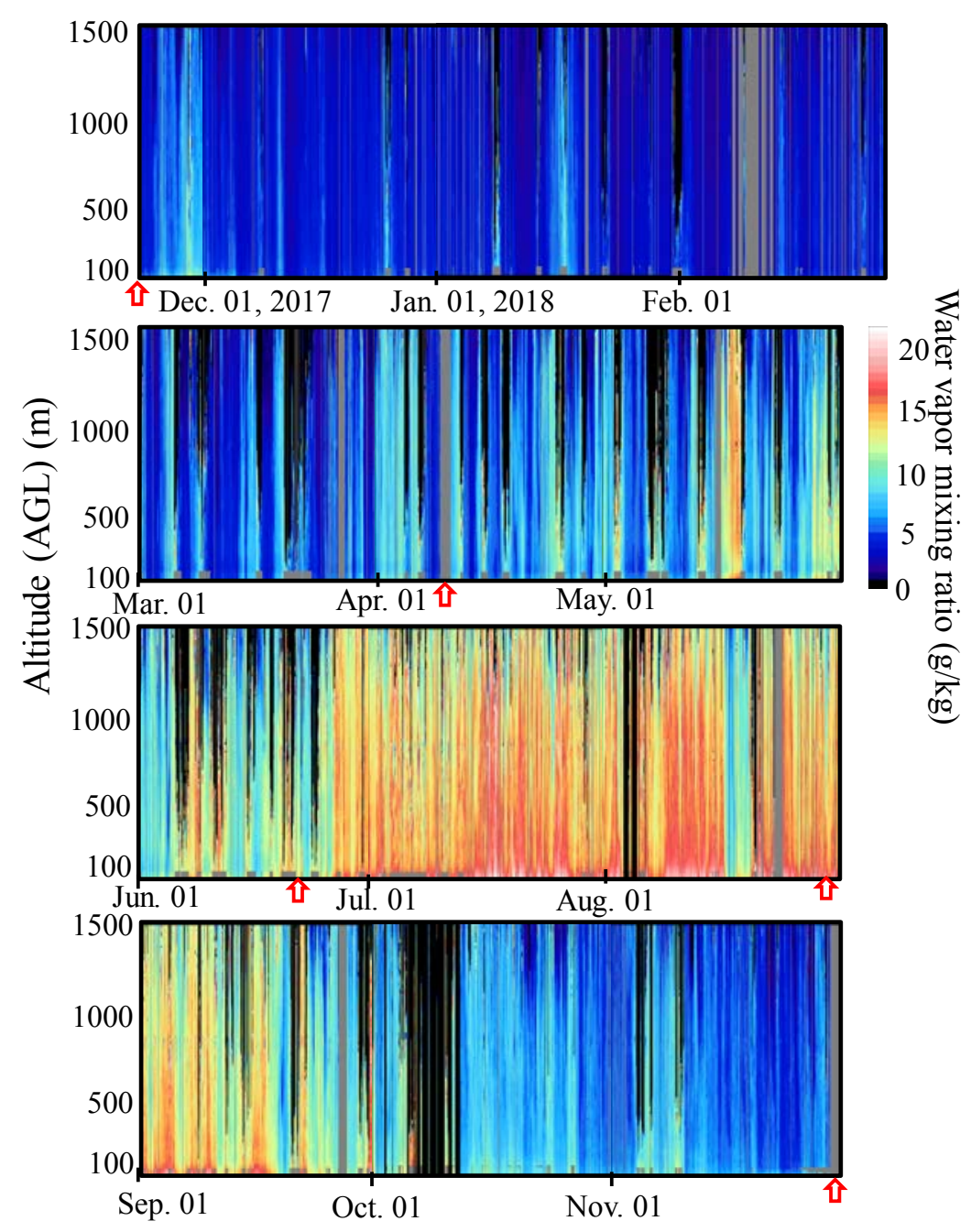

Figure 3. Time-height cross sections of the water vapor mixing ratio profiles observed by the Raman lidar at Shigaraki, Japan, from November 24, 2017, to November 29, 2018. Red arrows in x-axis correspond to the time of replacing the flash lamp of the laser.

\section{ACKNOWLEDGEMENTS}

A part of this study is supported by JSPS KAKENHI Grants 15H03724, 16H04999, and 26709042.

\section{REFERENCES}

[1] Whiteman, D., Melfi, S., and Ferrare, R., Raman lidar system for the measurement of water vapor and aerosols in the Earth's atmosphere, Appl. Opt., 31, 3068-3082 (1992).
[2] Renaut, D., Pourny, C., and Capitini, R., Daytime Raman lidar measurements of water vapor, Opt. Lett., 5, 232-235 (1980).

[3] Renault, D. and R. Capitini, Boundary-layer water vapor probing with a solar-blind Raman lidar: validations, meteorological observations and prospects, J. Atmos. Oceanic Technol., 5(5), 585- 601 (1988).

[4] Lazzarotto, B., M. Frioud, G. Larchevêque, V. Mitev, P. Quaglia, V. Simeonov, A. Thompson, H. van den Bergh, and B. Calpini, Ozone and water-vapor measurements by Raman lidar in the planetary boundary layer: error sources and field measurements, Appl. Optics, 40 (18), 2985- 2997 (2001). 